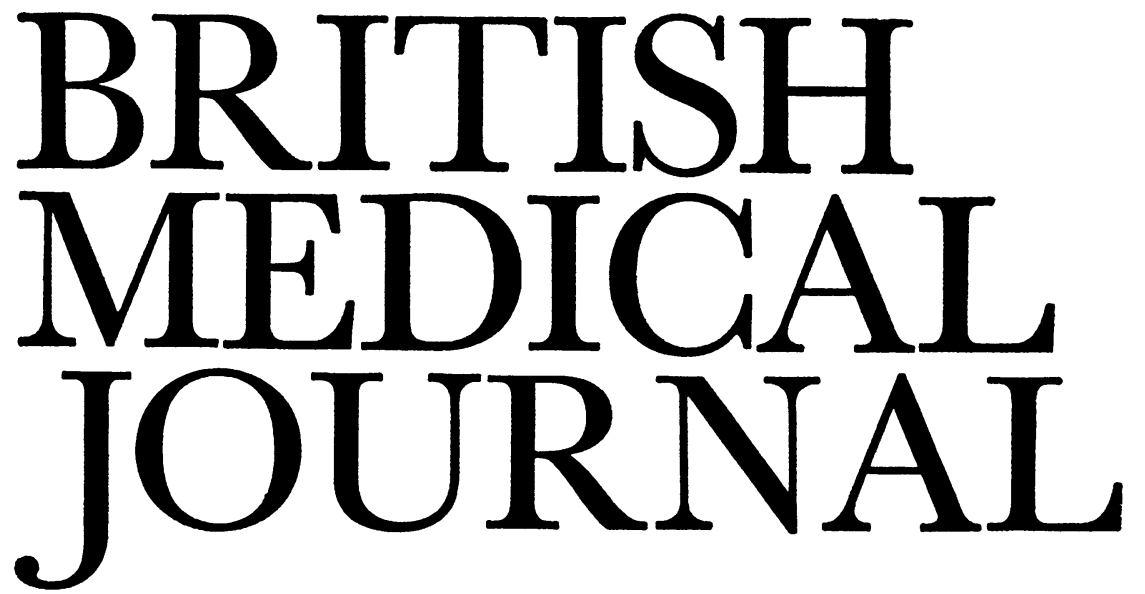

LONDON SATURDAY 6 OCTOBER 1973

\section{Are PUFA Harmful?}

Consumption of polyunsaturated fatty acids, or PUFA as we are learning to call them, has apparently substantially increased $^{1}$ over the two decades since H. M. Sinclair ${ }^{2}$ drew attention to their importance. Their beneficial effects in reducing the incidence of fatal ischaemic heart disease $\mathrm{e}^{34}$ have probably added to their popularity.

But it has been suggested that excessive consumption of PUFA carries certain risks, notably premature ageing and development of cancer. ${ }^{1}$ The evidence for premature ageing ${ }^{1}$ is tenuous. It depended on a greater declared consumption of PUFA by people appearing old for their years than by others appearing normal. Age was assessed somewhat subjectively by the extent of skin wrinkling, loss of hair, and suchlike. But people who appear old for their age, it could be argued, may be concerned with their old appearance and thus might be more generous with their dietary "polyunsaturates." Even if PUFA do cause degeneration of connective tissue, it would be interesting to know how this affects the onset of ischaemic heart disease. Senile dilatation (ectasia) of an artery might conceivably enlarge the bore faster than the inward growth of atherosclerosis tends to occlude it.

PUFA are characteristic of cell membranes with a fast turnover, ${ }^{5}$ unlike the more saturated sorts found in stable structures such as myelin. ${ }^{6}$ But this consideration is difficult to relate to premature ageing. It is perhaps more important that PUFA are liable, in the absence of antioxidants (such as vitamin E) to be oxidized to epoxides and hydroperoxides. ${ }^{7}$ These in turn polymerize to form ceroid and lipofuscin pigments. Lipofuscins in heart muscle, liver, and neurones seem to be harmless-indeed they play a part in the function of certain neurones ${ }^{8}$ - but ceroid is thought to be atherogenic. ${ }^{9}$ Peroxides depress tissue respiration, ${ }^{10}$ while 'the hydroperoxide free radical may damage tissues. ${ }^{11}$ The curious fact may be mentioned here that rabbits living in the wild on a remote barren Welsh island, and dependent on droppings from fish-eating birds for much of their diet, developed pigmentation and fibrosis of their adipose tissue. ${ }^{12}$ However, the rabbit is a very different animal from man, and there seems to be no well-recognized epidemiological evidence that large consumers of vegetable and fish oils (e.g., African, Japanese, and Eskimo populations) age more rapidly than communities relying on animal fats. It would be appropriate to know whether the Faroes Islanders, depending on a fishing industry, ${ }^{13}$ age more rapidly than their ethnically-similar neighbours, the agricultural and wool-producing Shetlanders. ${ }^{13}$
The risk of cancer developing in people consuming large amounts of PUFA has been more fully investigated. $M$. L. Pearce and S. Dayton ${ }^{3}$ found in Los Angeles an excess of cancers in people taking a PUFA-enriched prudent diet, even though these people suffered less ischamic heart disease than those consuming an ordinary diet. However, the excess of cancers was not statistically significant, and was not confirmed in a study in Finnish mental hospitals. ${ }^{4}$

The incidence of cancer is not increased in populations eating frsh and vegetable oils. Though cancer of the stomach is common in Japan, ${ }^{14} 15$ it is relatively uncommon in Africans ${ }^{15}$ and not noteworthy in Eskimos. ${ }^{16}$ Indeed the overall death rate from cancer in Africans, Japanese, and Eskimos is certainly not higher than in Europeans. ${ }^{15} 17$ However, comparisons with Caucasian statistics may be complicated by ethnic differences, peculiarities of diet, and differing life spans.

Though there seems to be little or no real risk of developing cancer from the prudent consumption of PUFA, some aliphatic epoxides-the early oxidation product of the unsaturated bond-are carcinogens, ${ }^{18}$ but these epoxides have not apparently been tested. Clearly the ingestion of epoxides carries potential risks. Nevertheless, it is not known whether epoxides are actually formed from unsaturated fats in significant amounts within the body apart from the classical sites of lipofuscin accumulation (heart muscle, liver, and neurones in the brain and spinal ganglia), nor is it certain how much vitamin $\mathbf{E}$ ( $\alpha$-tocopherol) is required in man to prevent such oxidation. Studies in animals suggest that the normal requirement is $2.4-3.2 \mathrm{mg}$ per 100 kilocalories of food per day, and this increases to $3.7-6.4 \mathrm{mg}$ with a high PUFA intake. ${ }^{19}$

Another way in which polyunsaturated cooking oils are oxidized is by repeated re-use and re-heating, such as was commonly practised in Great Britain during the war. It would be reasonable to regard this as a bad and possibly dangerous habit, whether owing to oxidation of PUFA or of some other constituent of cooking oils. ${ }^{20}$

No convincing case has been made that PUFA cause either premature ageing or cancer. Nevertheless, people consuming PUFA in quantity might consider taking supplements of vitamin $E$ and should bear in mind the view 2122 that saturated, monounsaturated, and polyunsaturated fatty acids should be consumed in equal amounts.

Concern about the possible danger of PUFA nearly fades into insignificance when compared with our ignorance about the fat composition of many widely consumed prepared foods. The term "vegetable oil" may include coconut oilbelieved to be a constituent of many ice-creams, "non- 
diary creamers," confections, and cooking oils. Yet coconut oil is $85 \%$ saturated, has a particularly low iodine-value, and contains large amounts of palmitic and lauric acids. ${ }^{23}$ Palmitic and myristic acids are regarded as particularly potent atherogenic agents, ${ }^{24}$ and the level of myristic acid is raised in the serum of patients with ischaemic heart disease. ${ }^{25}$ Its composition makes coconut oil an even less desirable component of the diet than dairy fats, beef-fat, and the like. If people are consuming more vegetable oils, they should insist on eating the right sorts-such as corn oil, soya-bean oil, or preferably sunflower-seed oil-and not one that is more saturated than "animal fat." Rapeseed oil, owing to its high content of erucic acid, is known to be cardiotoxic in animals, ${ }^{26}$ so that it would seem to be a most undesirable vegetable oil. But how are the public to know? They have a right to be informed about "vegetable oils." Manufacturers should declare the source and major composition of all fats used in foodstuffs. ${ }^{21} 22 \mathrm{~A}$ deplorable commercial secrecy envelops the topic of edible fats.

The public are also ignorant about the extent and effects of hydrogenating PUFA to make them more solid. This is reputed to cause trans-unsaturated fatty acids to be formed. The cholesterol esters of these fatty acids are known to be highly fibrogenic. ${ }^{27}$ Again the facts should be divulged. It is disturbing that we consume commercially processed foods without considering what they contain, how they are made, or what harm they may do.

1 Pinckney, E. R., American Heart Fournal, 1973, 85, 723.

2 Pinckney, E. R., American Heart 7nurna

3 Pearce, M. L., and Davton, S., I.ancet, 1971, 1. 464

4 Pearce, M. L., and Davton, S., L.ancet, 1971, 1. 464 . lainen, E., Lancet, 1972, 2, 835 .

5 Fleischer, S., and Rouser, G., Fournal of the American Oil Chemists' Society, 1965, 42, 588

O'Brien, J. S., Science, 1965, 147, 1099.

7 Granados, H., and Dam, H., Acta Pathologica Microbiologica Scandinavica, 1950, 27, 591 .

8 Friede, R. L., Acta Neuronathologica, 1962, 2, 113.

9 Hartroft, W. S., Fournal of Gerontology, 1953, 8, 158

10 Aoyama, S., and Iwakami, M., Fananese Heart fournal, 1965, 6, 128.

11 Tappel, A. L., Annals of the New York Academy of Sciences, 1972, 203, 12.

12 Jones, D., Gresham, G. A., Lloyd, H. G., and Howard, A. N., Nature, 1965, 207, 205.

Allison, R. S., Proceedings of the Royal Society of Medicine, 1963, 56, 71. Imai, T., Kubo, T., and Watanabe, H., Fournal of the National Cancer Institute, 1971, 47, 179

15 Dunham, L. I., and Bailar, J. C., fournal of the National Cancer Institute, $1968,41,155$

16 Fortuine, R., Cancer, 1969, 23, 468.

International Union Against Cancer, Cancer Incidence in Five Continents, Vol. 2, ed. R. Doll, C. Muir, and J. Waterhouse. Berlin, Springer, 1970. 18 Clayson, D. B., Chemical Carcinogenesis. London, Churchill, 1962.

19 Jager, F. C., Annals of the New York Academy of Sciences, 1972, 203, 199. Symposium: Biological Significance of Autoxidized and Polymerized Oils, Lipids, 1973, 8, 329 .

21 Atherosclerosis Study Group, Circulation, 1970, 42, A-55.

22 Fredrickson, D. S., British Medical fournal, 1971, 2, 187.

23 Thorpe, J. F., and Whiteley, M. A., Thorpe's Dictionary of Applied Chemistry, Vol. 3, p. 241. London, Longmans, 1939.

24 Kritcehvsky, D., and Tepper, S. A., Progress in Biochemical Pharmacology 1968, 4, 474 Symposium: Recent Advances in Atherosclerosis, cology, 1968, 4, 474. Symposium: Recent Advances in Atherosel. Basel.

25 Colehour, J. K., and Leonard, R. H., Clinica Chimica Acta, 1964, 9, 413.

25 Colehour, J. K., and Leonard, R. H., Clinica Chimica

27 Abdellatif, A. M. M., Nutrition Reviews, 1972, 30, 2 . ology and Bacteriology, 1967, 94, 63.

\section{Diagnosis of Childhood Spinal Muscular Atrophy}

A severe form of spinal muscular atrophy developing at or soon after birth and leading to hypotonia and progressive muscular weakness is known as Werdnig-Hoffmann disease. A more benign form is now recognized as being relatively common in childhood and adolescence. E. Kugelberg and L.
Welander ${ }^{1}$ were among the first to define this syndrome, which is sometimes called "pseudomyopathic spinal muscular atrophy." Controversy persists on whether it represents a distinct clinical and genetic entity or the benign end of a range of disease with severe Werdnig-Hoffmann disease at the other end. Severe and relatively benign cases may sometimes be seen in the same sibship, ${ }^{2-4}$ but there is growing evidence $^{5}$ to indicate that despite such atypical families spinal muscular atrophy of childhood contains at least three subvarieties which are clinically and genetically distinct. The first of these, it is suggested, is classical and severe WerdnigHoffmann disease, with onset at birth or within the first few months of life and with death before the age of 2 years. In the second, more chronic, category the onset is usually later within the first year of life, and many patients survive, though severely disabled, for five to 10 years or even longer, though they are never able to walk. The third category, corresponding to the cases described by Kugelberg and Welander, comprises patients in whom muscular weakness becomes apparent later, within the first few years of life. Most of them are able to walk, though the rate of increase in their disability and of the progression of the disease is variable. While occasional families show $\mathrm{X}$-linked recessive or dominant inheritance, most cases of spinal muscular atrophy in childhood probably result from an autosomal recessive gene, though many continue to occur sporadically. The question whether the recessively inherited cases can indeed be classified into the three categories defined by $K$. Fried and A. E. H. Emery ${ }^{5}$ remains open, and an international collaborative study designed to answer it has recently been launched. 6

Diagnostic difficulties may arise in distinguishing between benign spinal muscular atrophy of the Kugelberg-Welander type on the one hand and Duchenne type muscular dystrophy on the other. These difficulties are heightened by the knowledge that substantial increases in the activity of creatine kinase in the serum, as well as electromyographic abnormalities sometimes suggesting a primary myopathy, may be observed in some of these cases, ${ }^{7}$ and even muscle biopsy does not invariably give conclusive findings, especially if histochemical techniaues of examining muscle sections are not available. So-called secondary myopathic change ${ }^{78}$ in muscle sections may obscure the underlying neurogenic nature of the condition, though histochemical type-grouping of muscle fibres may be a useful clue to the primary neurogenic character of the disease.

A. Moosa and V. Dubowitz ${ }^{9}$ have now drawn attention to two useful pieces of clinical evidence which may help in distinguishing cases of childhood spinal muscular atrophy. They note that there have been occasional previous reports $^{3}{ }^{10-13}$ of patients with spinal muscular atrophy who showed tremor of the limbs. And in the past three years they have seen 13 patients with spinal muscular atrophy of the benign childhood type all with coarse irregular tremor of the bands, particularly when the arms were held outstretched with the fingers splayed. In addition, they have noted that many patients with spinal muscular atrophy tend to walk flat-footed with eversion of the feet, while patients with typical Duchenne muscular dystrophy tend to walk on their toes, with evidence of an equinovarus type of foot deformity. Clearly therefore these signs should be sought when difficulty arises in distinguishing between benign spinal muscular atrophy and Duchenne muscular dystrophy in childhood. As Duchenne dystrophy is invariably a disease of males, diagnostic difficulty is less when the affected child is 